\title{
Fonction occlusale
}

\section{de la canine en implantologie}

\section{Occlusal function of the canine tooth in implantology}

\section{Résumé}

MOTS-CLEFS :
- Implant, occlusion,
neurophysiologie,
canine
KEYWORDS:
- Implant, occlusion,
neurophysiology,
canine

$\operatorname{AOS} n^{\circ} 273-2015$
Le remplacement d'une canine par un implant va, au niveau de la neurophysiologie, remplacer un système de protection basé sur la présence de récepteurs desmodontaux par un système ankylosé dans l'os et ne transmettant que des informations faibles et filtrées aux noyaux sensitifs supérieurs. La présence ou non de récepteurs antagonistes va orienter le choix du concept occlusal à appliquer, avec comme règle première que plus le concept est simple, mieux il sera réalisé.

\author{
Abstract
}

The replacement of a canine tooth by an implant will, in its neurophysiologic consequences, exchange a protection system based on the presence of periodontal membrane receptors with one that depends on an implant ankylosed in bone that can transmit only feeble information to be filtered by sensitive superior ganglia. The presence or absence of antagonistic receptors will guide the practitioner in selecting which occlusal concept to apply, with the first rule being that the simpler the concept, the more successfully it can be accomplished.

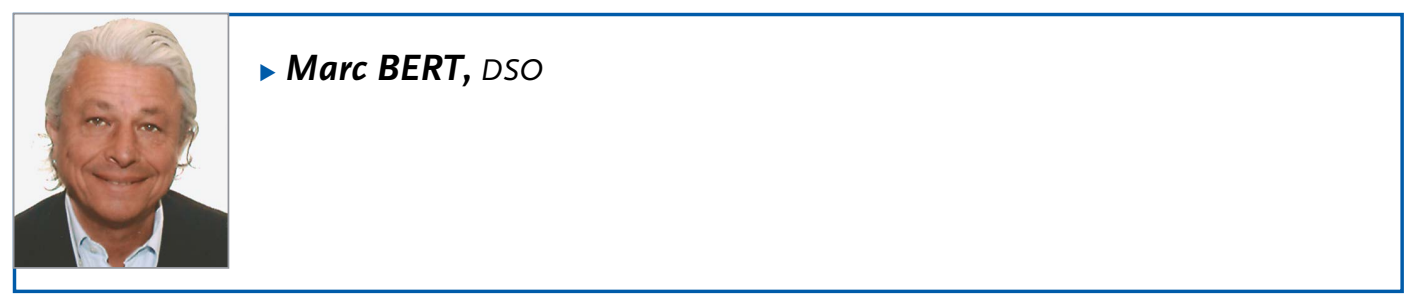

\section{INTRODUCTION}

Les muscles élévateurs et la plupart des muscles abaisseurs ont leur innervation motrice commandée par le nerf trijumeau, $\mathrm{V}^{\circ}$ paire crânienne. Cette innervation motrice est commandée par un noyau moteur dépendant lui-même d'un noyau sensitif intégrant une multitude d'informations parmi lesquelles celles issues des pressions sur les dents, pressions captées par des récepteurs situés dans le desmodonte de la dent. C'est la proprioception. Ces « capteurs » vont induire les différentes réactions de la mandibule, y compris les positions pathologiques que la compréhension de l'anatomie et de la physiologie de l'ATM, des muscles et de leurs rapports vont permettre bien évidemment de traiter. Les particularités occlusales des prothèses sur implants viennent du fait qu'il n'existe pas de récepteurs desmodontaux autour des implants qui sont ankylosés dans l'os. La canine joue un rôle majeur dans la plupart des concepts occlusaux par son influence sur les mouvements de la mandibule. De nombreuses questions peuvent se poser lorsqu'elle est remplacée par un implant.

\section{LA PROPRIOCEPTION}

La proprioception est la fonction permettant au système nerveux central d'être renseigné sur la position d'un muscle ou d'une articulation et sur les pressions qui éventuellement s'y exercent. Dans le cas d'une dent, la proprioception renseigne les centres nerveux supérieurs sur les pressions subies par les dents, ce qui permet à ces centres de moduler la contraction des muscles manducateurs : 
I soit en augmentant la pression afin d'écraser un aliment résistant ;

I soit en inhibant la pression si celle-ci dépasse des limites acceptables par l'organisme.

L'origine de la proprioception dentaire est le récepteur desmodontal situé dans le ligament alvéolo-dentaire (Fig. 1).

Les récepteurs desmodontaux sont (Woda, 1983) :

$\checkmark$ adaptables ou non adaptables. Il s'agit soit de terminaisons libres, soit de récepteurs plus fins, encapsulés ou non ;

I sensibles à la vitesse d'application de la stimulation. Les forces peuvent être appliquées graduellement ou instantanément, et sont captées par des récepteurs spécifiques ;

I soumis à un seuil qui est de l'ordre de 1 gramme ;

I sensibles à l'intensité de la force appliquée et capables de détecter de très faibles variations de l'amplitude de la stimulation ;

capables d'une sensibilité directionnelle.

Les récepteurs desmodontaux sont en grand nombre pour les incisives et canines, et leur nombre va en décroissant des prémolaires aux molaires. Ils enregistrent les pressions exercées sur une dent, qu'elles soient axiales ou latérales, et transmettent ces pressions au noyau sensitif du trijumeau (cinquième paire crânienne ou V) par l'intermédiaire d'un premier neurone (ou protoneurone) dont le corps cellulaire se situe dans le ganglion de Gasser (Fig. 2).

L'information circule :

par la branche maxillaire du trijumeau, ou V2, pour les dents maxillaires ;

I par la branche mandibulaire du trijumeau, ou V3, pour les dents de l'arcade inférieure.

La branche V1, l'ophtalmique de Willis, ne transmet aucune information d'origine dentaire.

En simplifiant à l'extrême, l'information perçue par le noyau sensitif du trijumeau est transmise à 2 noyaux différents, en fonction de l'intensité des pressions enregistrées par le récepteur desmodontal :

$\checkmark$ le noyau moteur du V, qui déclenche la contraction des muscles élévateurs pour augmenter leur action afin d'écraser un aliment (Fig. 3) ;

$\checkmark$ le noyau inhibiteur du V (noyau supratrigéminal), si la pression dépasse certaines limites physiologiques (Fig. 4). La contraction musculaire est alors stoppée, avec parfois contraction des muscles abaisseurs en cas de choc sur la dent.

Le seuil d'apparition d'une réponse à une stimulation mécanique est très bas et a été estimé à 1 gramme par Woda et Fontenelle (1975) et Woda (1983). Cette sensibilité a été qualifiée par ces auteurs d'épicritique et de directionnelle. Une sensibilité épicritique est fine et transmise par des fibres nerveuses de gros calibre,

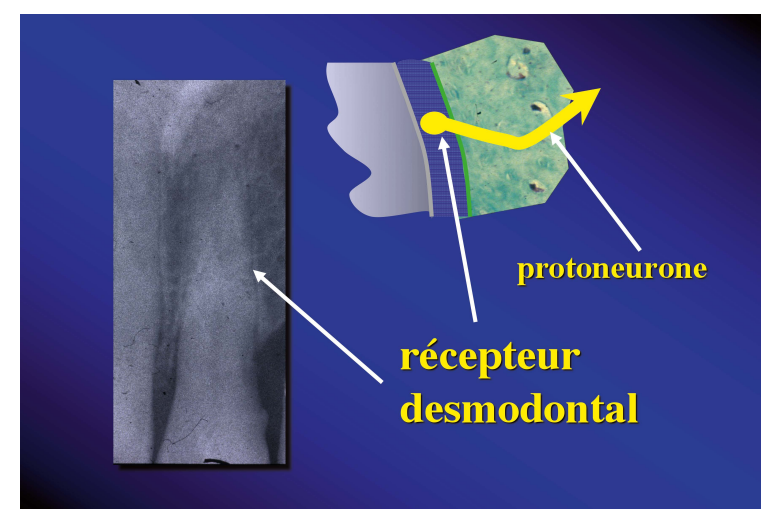

$\triangle$ Fig. 1 : Le ligament alvéolo-dentaire contient un nombre très élevé de récepteurs desmodontaux.

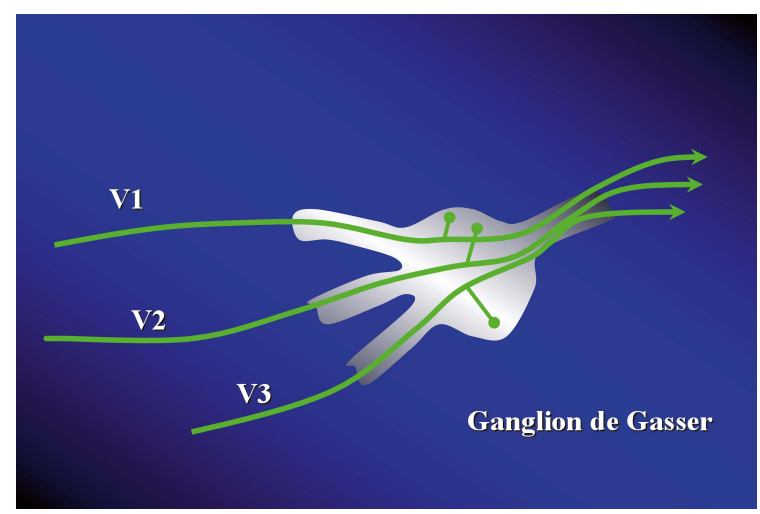

$\triangle$ Fig. 2 : Les informations captées par les récepteurs desmodontaux maxillaires et mandibulaires passent par le ganglion de Gasser (siège du corps cellulaire des neurones) et arrivent sur le noyau sensitif du trijumeau.

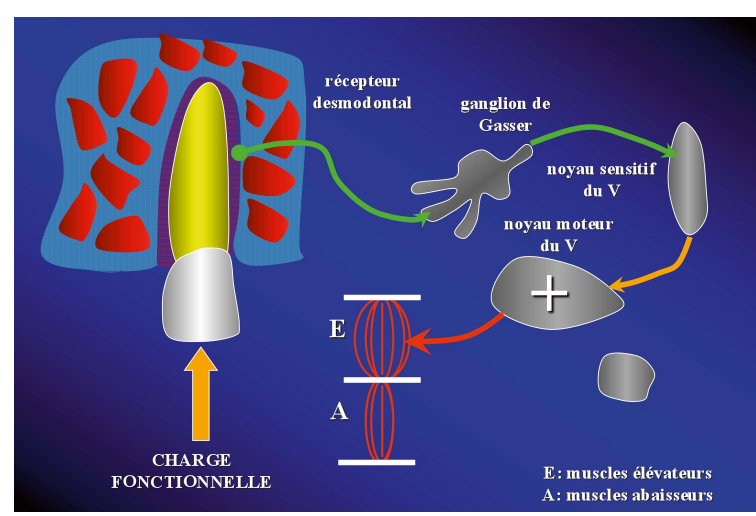

$\triangle$ Fig.3 : Lorsque la pression est dans la limite acceptable des charges, le noyau sensitif du trijumeau « commande " l'activation des muscles élévateurs par l'intermédiaire du noyau moteur du trijumeau (vert : sensitif; orange : interneurone; rouge : moteur).

très myélinisées et ne perdant aucune information. La sensibilité épicritique d'origine desmodontale est directionnelle car le patient testé est capable d'indiquer si la force appliquée l'est en pression ou en traction, confirmant ainsi sa finesse de perception.

En résumé, le parodonte est à l'origine d'une activité neuro-physiologique très fine et très discriminative, mettant en jeu un mécanisme protecteur très organisé, à partir d'un seuil très bas, faisant dire que la dent est une branche du trijumeau. 


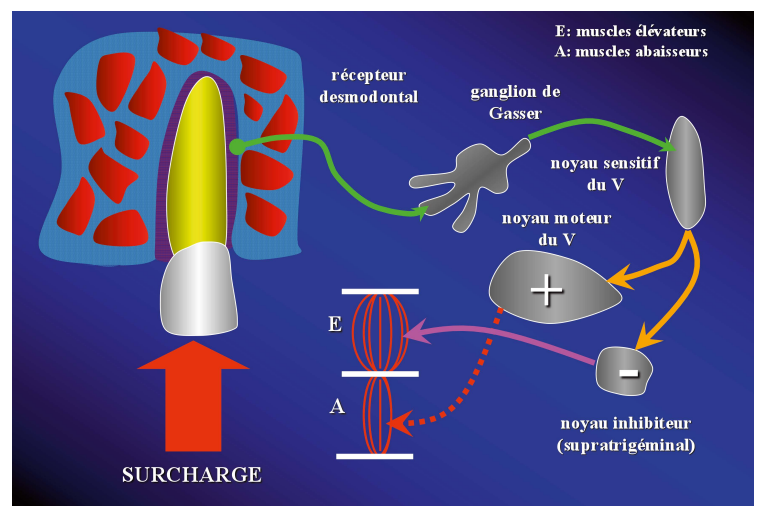

$\triangle$ Fig.4 : Lorsque la pression se situe au-delà de la limite acceptable des charges, le noyau sensitif déclenche l'arrêt de la contraction des muscles élévateurs par l'intermédiaire du noyau inhibiteur du trijumeau, et parfois active la contraction des muscles abaisseurs de la mandibule (en pointillé) (vert : sensitif ; orange : interneurone ; violet : inhibiteur; rouge : moteur).

\section{LES « CONCEPTS » OCCLUSAUX}

La reconstruction d'une bouche s'appuie sur des conceptions permettant aux articulations temporo-mandibulaires et aux muscles qui l'actionnent de «fonctionner » dans une enveloppe physiologique de mouvements (Bert, Leclercq, 2015).

\section{En position d'intercuspidie maximale}

Deux principes vont guider la reconstruction prothétique en position d'intercuspidie maximale :

\1- trouver ou retrouver une position d'intercuspidie correspondant à une absence de contraction des muscles ptérygoïdiens latéraux, principaux responsables de la plupart des pathologies occlusales (Bert, Leclercq, 2015);

I 2 - obtenir une stabilité des contacts.

\section{En propulsion}

Lors de la propulsion, le glissement des incisives mandibulaires sur la face palatine des incisives maxillaires doit entraîner instantanément la désocclusion des dents cuspidées (aidé en cela par la courbe de Spee). C'est le « guide antérieur ». Le réglage du guide antérieur est facilité par la faible amplitude en latéralité du mouvement de propulsion (Fig. 5), permettant d'obtenir un guidage satisfaisant dans l'ensemble des mouvements de glissement antérieur.

\section{En latéralité}

Plusieurs conceptions de la latéralité ont été proposées: fonction de groupe, occlusion «balancée " et fonction canine, conceptions qu'il faut apprécier à l'aune de leur intérêt théorique et de leur «réalisibilité ", c'est-à-dire de leur application correcte en bouche.

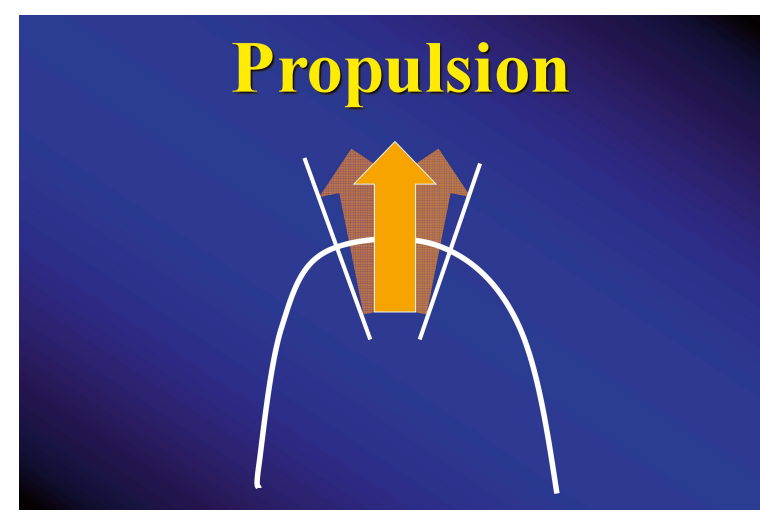

$\triangle$ Fig. 5 : Le mouvement de propulsion présente une faible amplitude en latéralité, facilitant en clinique le réglage de ce mouvement. Dès qu'une canine est intéressée, ce mouvement devient une latéralité.

$\checkmark 1$ - La fonction de groupe. En théorie, c'est le concept idéal, plusieurs groupes de dents assurant le guidage de la mandibule du côté travaillant, répartissant les charges sur plusieurs éléments au lieu d'un seul. En pratique, il existe cependant quelques écueils :

- il n'existe pas une seule latéralité, mais une infinité de latéralités (Fig. 6) ;

Vouloir, dans chacun de ces mouvements, obtenir des contacts fonctionnels est extrêmement difficile, voire impossible, en sachant qu'une erreur d'un degré sur une pente cuspidienne peut transformer un contact stabilisant en interférence. De plus, le stress accumulé au cours de la journée fait que la position de la mandibule n'est pas la même le matin et le soir, compliquant encore plus la situation (Fig. 7 à 9) ;

- cette conception oblige à raccourcir la canine (Fig. 10), ce qui a un impact non négligeable sur l'esthétique du sourire, d'autant plus si la canine controlatérale conserve une longueur normale (Fig. 11, 12).

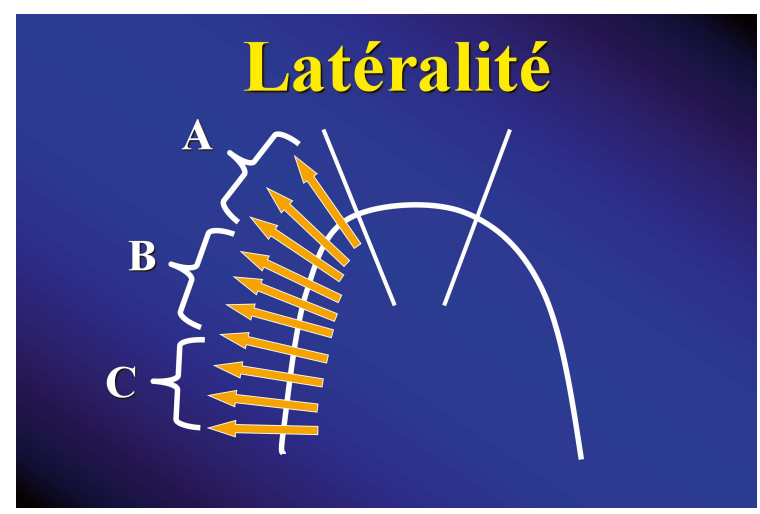

$\triangle$ Fig. 6 : Il existe une infinité de latéralités, alliant au mouvement latéral simple (B) plus ou moins de propulsion (A) ou de rétropulsion $(\mathrm{C})$. La fonction de groupe nécessite des glissements parfaits dans chacune de ces positions, ce qui semble difficile, sauf en théorie... 


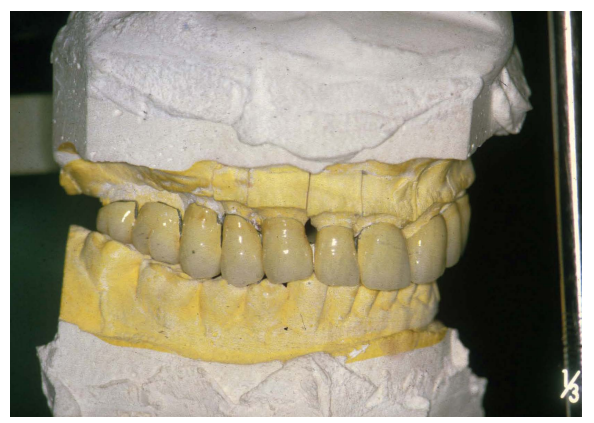

$\triangle$ Fig. 7 : Un articulateur correctement programmé permet d'obtenir des contacts en glissement latéral sur plusieurs groupes de dents.

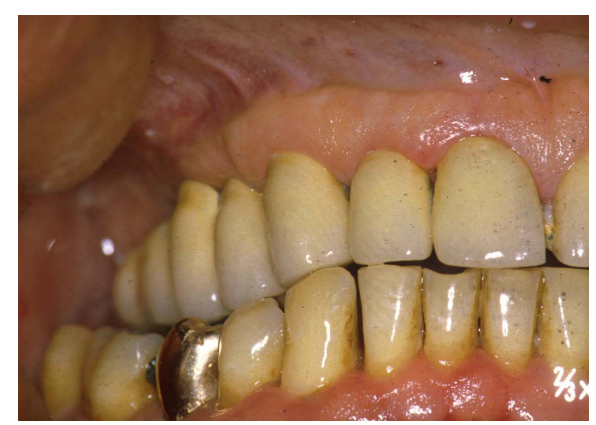

$\triangle$ Fig. 8 : En bouche, le contrôle effectué un matin montre que des contacts de groupe en latéralité peuvent être obtenus.

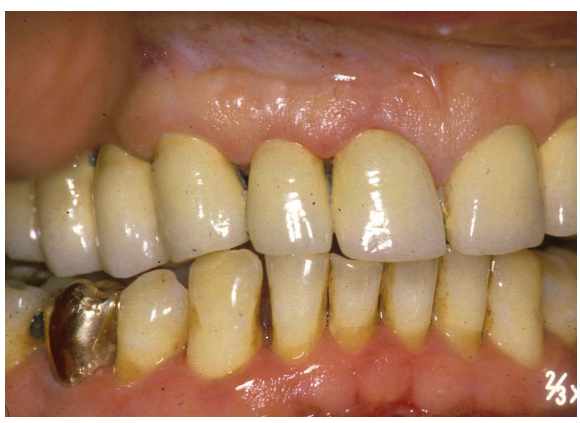

Fig. 9 : Par contre, le soir, la position de la mandibule n'est plus la même et la trajectoire en latéralité est totalement modifiée.

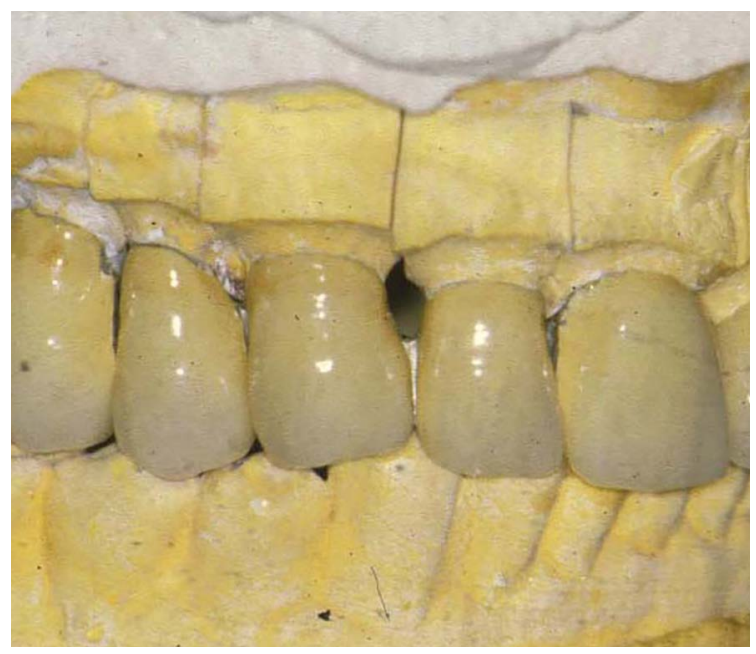

$\triangle$ Fig. 10 : Un gros plan de la figure 7 montre que la canine doit être raccourcie pour permettre la fonction de groupe, ce qui est esthétiquement très discutable, mais acceptable dans ce cas car les 2 canines ont subi le même traitement.

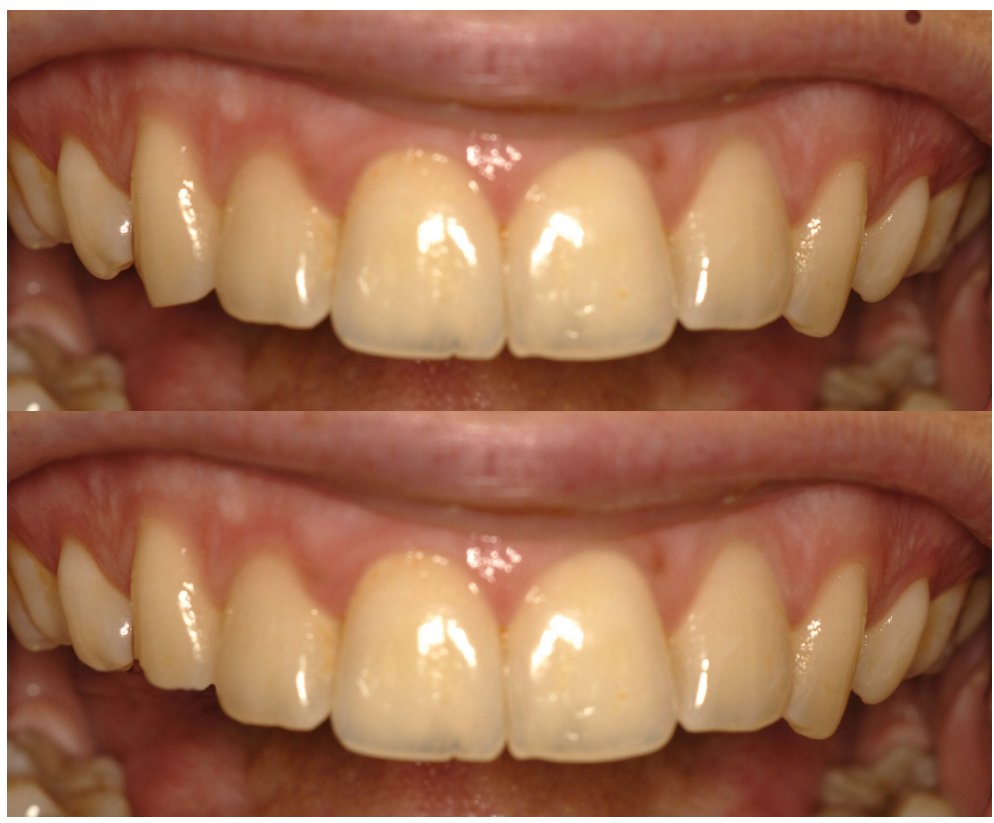

$\triangle$ Fig. 11 : Le sourire montre, dans ce cas, des canines de bonne longueur et équilibrées. Fig. 12 : La canine droite a été retouchée (merci Photoshop...) afin de permettre une fonction de groupe de ce côté, rendant inesthétique le sourire par la différence de longueur des 2 canines.
I 2 - La fonction balancée. Cette conception, mise au point en prothèse adjointe complète, permet d'obtenir la stabilisation de prothèses amovibles totales dans toutes les excursions mandibulaires. Dans le mouvement de propulsion, il existe des contacts postérieurs afin de stabiliser la prothèse maxillaire et éviter sa désinsertion postérieure. En latéralité, une fonction de groupe est construite du côté travaillant et il est conservé un ou des glissements stabilisants du côté non travaillant, toujours afin d'éviter le basculement des prothèses amovibles. L'obtention de ces contacts est difficile à obtenir (et souvent l'œuvre de spécialistes), les défauts de construction au niveau des pentes cuspidiennes étant compensés par la dépressibilité de la muqueuse d'appui qui permet une certaine marge d'erreur. La transposition de cette conception de l'occlusion en prothèse fixe est illusoire car il n'existe aucune marge d'erreur possible, en particulier pour les contacts stabilisants du côté non travaillant qui sont le plus souvent transformés en interférences, particulièrement destructrices.

$\checkmark 3$ - La fonction canine. Dans ce concept, le mouvement de latéralité entraîne une désocclusion immédiate des groupes cuspidés, du côté travaillant et du côté non travaillant, par le glissement de la canine inférieure sur la face palatine de la canine supérieure (Fig. 13, 14).

Ce concept simple ne fait intervenir que 4 dents, les canines dont on a vu qu'elles étaient très richement dotées en récepteurs desmodontaux, permettant de moduler la contraction des muscles manducateurs. Un articulateur simple permet de construire facilement ce glissement qui est vérifié en bouche avec les prothèses d'attente, puis avec les prothèses d'usage (Bert, Leclercq, 2015).

D'une manière routinière, le concept de la fonction canine permet de construire des prothèses fonctionnelles, simples (donc bien réalisées...) et bénéficiant 


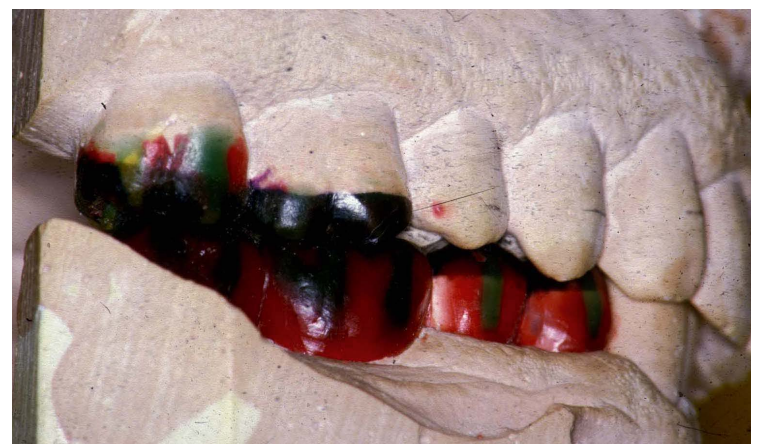

$\triangle$ Fig. 13 : Sur un articulateur, la stabilité des contacts en position d'intercuspidie maximale est vérifiée.

de la sécurité apportée par la présence de récepteurs desmodontaux en grand nombre sur la canine, permettant un excellent guidage neuromusculaire du mouvement tout en évitant les surcharges occlusales destructrices pour les dents, le parodonte ou les articulations temporo-mandibulaires liées aux interférences (aidé en cela par les courbes de Wilson).

\section{INTÉGRATION \\ NEUROPHYSIOLOGIQUE D'UN IMPLANT DENTAIRE}

On l'a vu plus haut, les récepteurs desmodontaux d'une dent sont sensibles à des pressions dont le seuil est d'un gramme et donnent des informations épicritiques (fines, discriminatives, véhiculées par des fibres de gros diamètre très myélinisées) et directionnelles (la direction du stimulus est perçue).

Qu'en est-il au niveau d'un implant dentaire (Fig. 15) ? En d'autres termes, quel est le devenir du récepteur desmodontal et de son protoneurone après l'extraction d'une dent ? Est-il totalement détruit ? Se transforme-t-il en une terminaison nerveuse plus simple : fibre ultraterminale, terminaison libre (nociceptive)? Est-il intégralement conservé ?

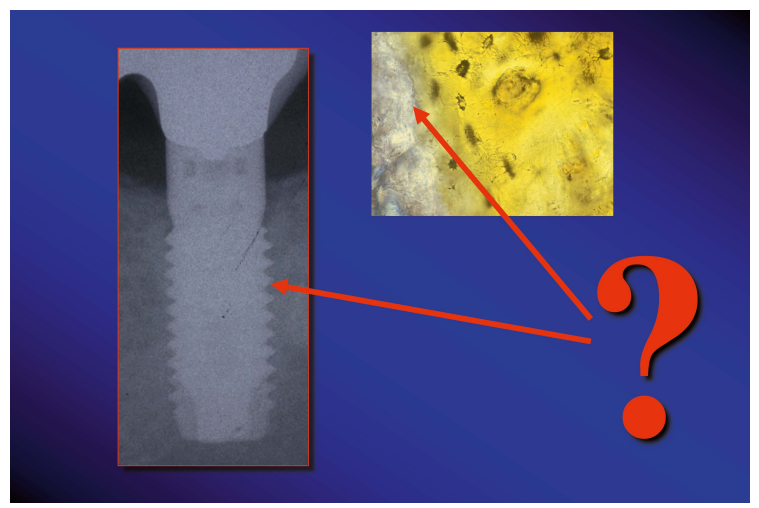

$\triangle$ Fig. 15 : Un implant est ostéo-intégré, c'est-à-dire ankylosé dans l'os, sans aucune structure ligamentaire.

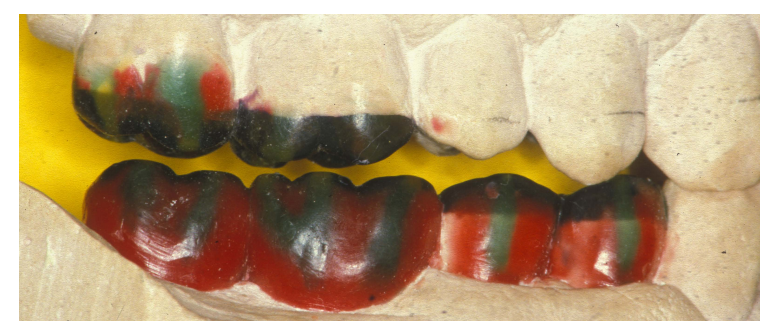

$\triangle$ Fig. 14 : En latéralité, le glissement de la canine inférieure sur la face palatine de la canine supérieure permet d'obtenir une désocclusion immédiate des groupes cuspidés, du côté travaillant et du côté non travaillant.

\section{Expérimentation clinique}

L'expérimentation clinique simple permet d'apporter des réponses à ces interrogations. À partir de 1981, et publiée en 1987 (Bert, 1987), une étude a été menée sur 50 implants de type lame de Linkow ou vis de Cherchève, c'est-à-dire non-enfouissables, donc fibro-intégrés. Le patient est initialement "éduqué » sur une dent naturelle présente en bouche, lui permettant de comprendre les informations attendues de lui. Un dynamomètre sensible au gramme (Fig. 16, 17) est connecté à une dent par l'intermédiaire d'une tige métallique. Le seuil de 1 gramme est généralement retrouvé sur cette dent. Le même dispositif est ensuite placé sur un implant, et le seuil est proche de 8 grammes, avec une faible variabilité ( 7 à 9 grammes). La même expérimentation a été reprise à partir de 1991 sur 50 implants ostéo-intégrés, et des résultats identiques ont été trouvés : un seuil de 8 grammes en moyenne avec une variabilité de 7 à 9 grammes (Bert, 2009). Une récente méta-analyse de Higaki et al (2014) a montré que les seuils de sensibilité au niveau des implants étaient d'environ 4 à 20 fois plus élevé que ceux d'une dent, avec une différence moyenne standardisée
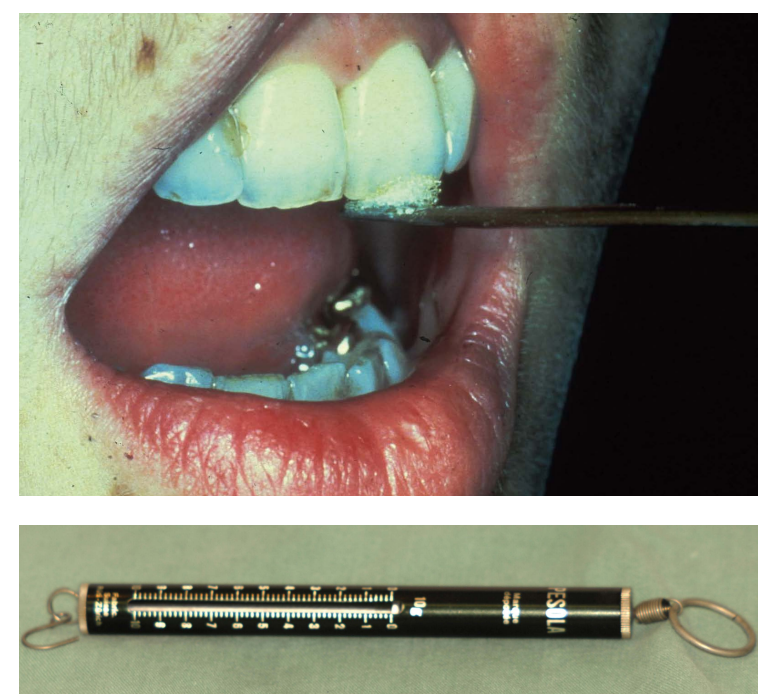

$\triangle$ Fig. 16 et 17 : Un dynamomètre sensible au gramme est connecté à une dent, puis à un implant à l'aide d'une tige métallique. 
(DMS) de 8,36, très proche des résultats publiés en 1987 et 2009... Mais dans tous les cas, la sensation a été décrite par les patients comme étant vague, diffuse, ce que les physiologistes qualifient comme étant « protopathique ", c'est-à-dire véhiculée, comme pour la douleur diffuse, par des fibres de faible calibre, pas ou peu myélinisées, et surtout soumises à une filtration. Plusieurs théories ont été émises pour expliquer cette filtration comme celle de Noordenbos, au niveau de la première synapse (Fig. 18), ou celle actuellement plus en vogue $\mathrm{du}$ « gate control» de Wall et Melzack (Fig. 19).

Quelle que soit la théorie retenue, il est fondamental de comprendre que les informations reçues par les structures péri-implantaires semblent être véhiculées par des voies qui sont soumises à un mécanisme de régulation au niveau de leur première synapse, alors que les afférences desmodontales ne sont pas soumises à ce mécanisme. Et si toutes les informations desmodontales, même les plus minimes, déclenchent un mécanisme protecteur qu'un praticien «occluso-conscient » saura détecter, il n'en est pas de même avec les implants dentaires, car seules les informations les plus importantes sont acheminées aux centres supérieurs, ce qui est aujourd'hui nommé : « ostéoperception».

\section{Conséquences cliniques}

La surcharge occlusale sur une dent déclenche le mécanisme protecteur décrit plus haut. L'absence de proprioception sur un implant ne met pas en jeu ce mécanisme, et la surcharge se maintient. Selon les lois de Wollf (1892), la réponse osseuse va dépendre de l'intensité de la charge : renforcement de l'os si elle est dans les limites physiologiques, destruction de l'os lorsqu'elle dépasse ces limites physiologiques (Fig. 20). D'autres fois, la surcharge occlusale non contrôlée entraîne la fracture d'un ou de plusieurs implants, de vis de prothèse, d'armatures métalliques et/ou de prothèses antagonistes.

Cette volonté de protéger l'implant de toute surcharge occlusale a conduit de nombreux auteurs à émettre des notions, hélas répandues, sur les concepts occlusaux à adopter sur les implants dentaires, comme la mise systématique des prothèses en sous-occlusion ou la fonction de groupe :

I la mise en sous-occlusion. Il a souvent été proposé de mettre la couronne sur implant en « légère sous-occlusion ", ou bien de n'autoriser les contacts sur un implant qu'en occlusion serrée du fait de l'absence de résilience d'un implant par rapport à une dent. Ces propositions traduisent une méconnaissance totale de la physiologie d'une dent et du comportement biomécanique d'un implant (Bert, Leclercq, 2015, chapitre 11). À partir du moment où la dent

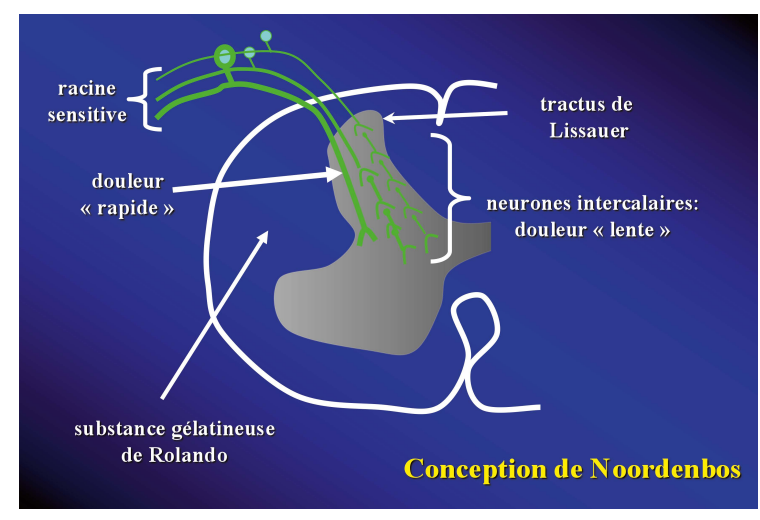

$\triangle$ Fig. 18 : La théorie de Noordenbos (1959) fait jouer au seul agencement multisynaptique de la première synapse un rôle inhibiteur pour les fibres de petit diamètre (d'après Mamo, 1968).

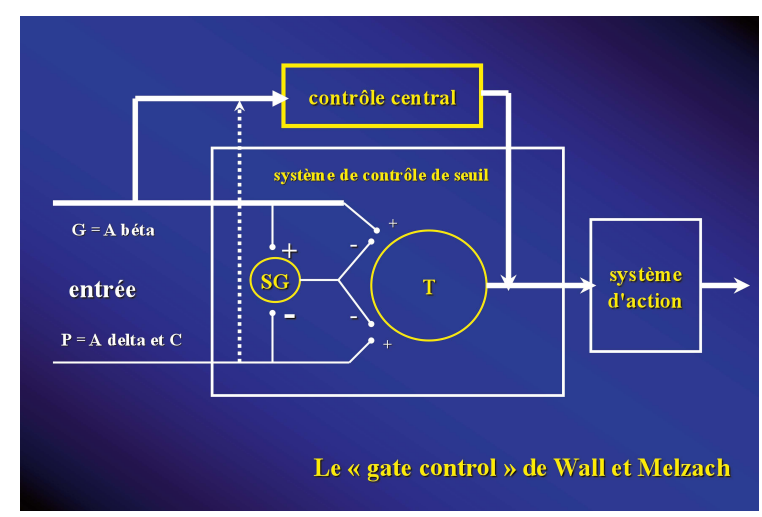

$\triangle$ Fig. 19 : La théorie de Wall et Melzack (1965) fait intervenir le contrôle central par des fibres de gros diamètre, contrôle autorisant ou non le passage des informations protopathiques (d'après Mamo, 1968).

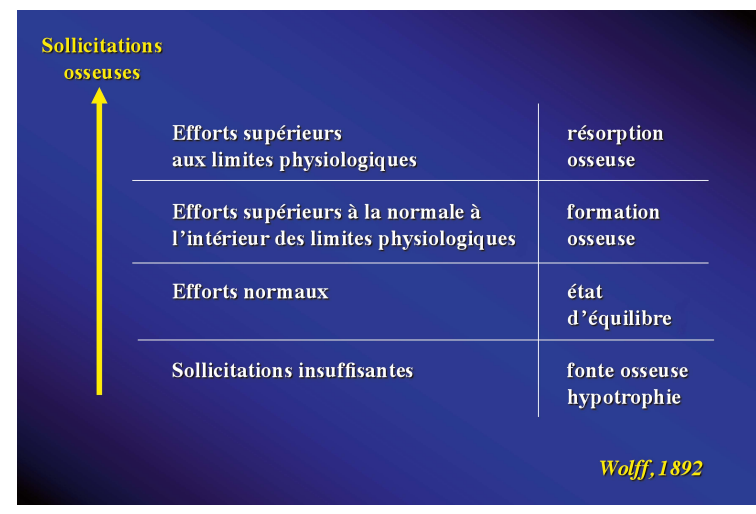

$\triangle$ Fig. 20 : Les lois de Wollf, maintes fois vérifiées, indiquent la réponse de l'os en fonction de l'intensité de la charge que celui-ci subit, l'insuffisance de charges ou la surcharge causant une résorption alors que des charges physiologiquement acceptables entraînent le maintien ou le renforcement de l'os.

antagoniste à l'implant est en sous- occlusion, elle subit une égression physiologique lui permettant de retrouver un calage occlusal nécessaire à sa stabilité. Et ce calage occlusal maintient la dent en place à chaque intercuspidie liée par exemple à la déglutition à vide (1500 à 2000 fois par jour !), l'empêchant d'égresser. Si l'implant est mis en sous-occlusion, la dent antagoniste va égresser afin de retrouver son nécessaire calage occlusal, avec un rapport qui n'a 


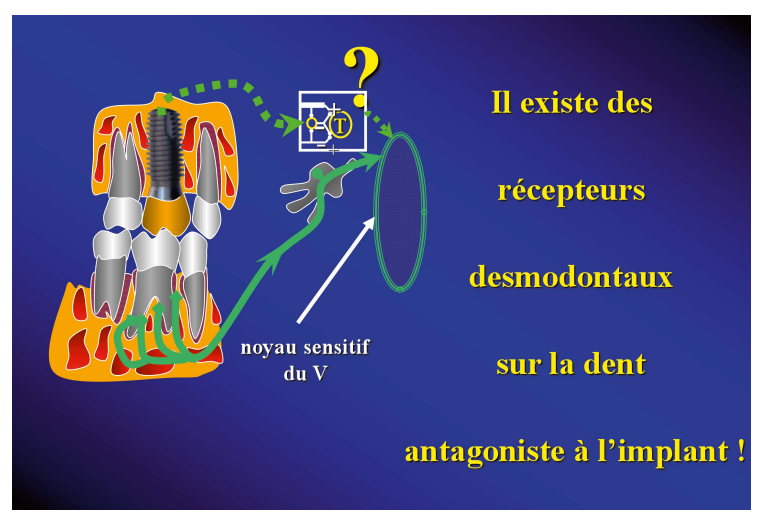

$\triangle$ Fig. 21 : Lorsque l'implant fait face à une dent, celle-ci, munie de récepteurs desmodontaux, prend en charge la proprioception et la régulation de la contraction des muscles manducateurs.

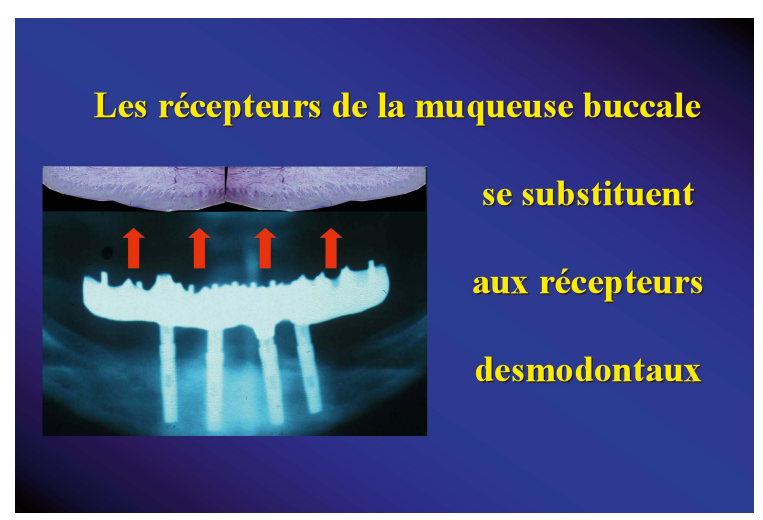

$\triangle$ Fig. 22 : Lorsque l'implant fait face à une selle prothétique, les récepteurs de la muqueuse buccale sont suffisamment discriminatifs pour prendre en charge une proprioception valable.

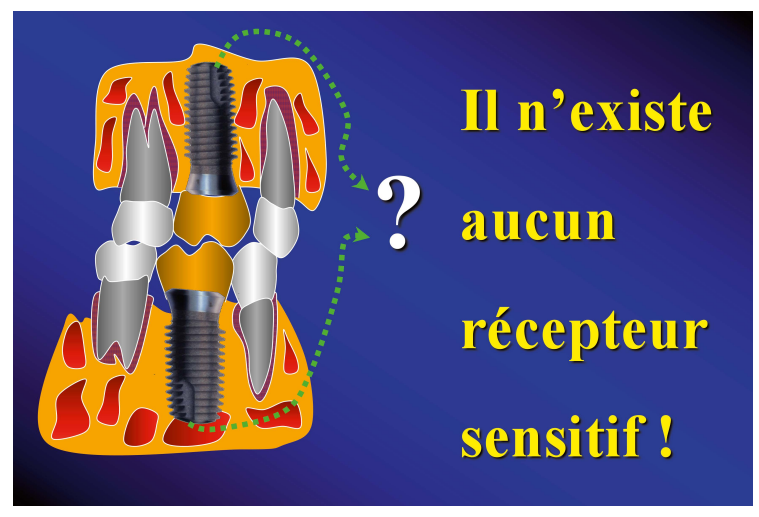

$\triangle$ Fig. 23 : Lorsque 2 implants sont face à face, il n'existe aucune proprioception.

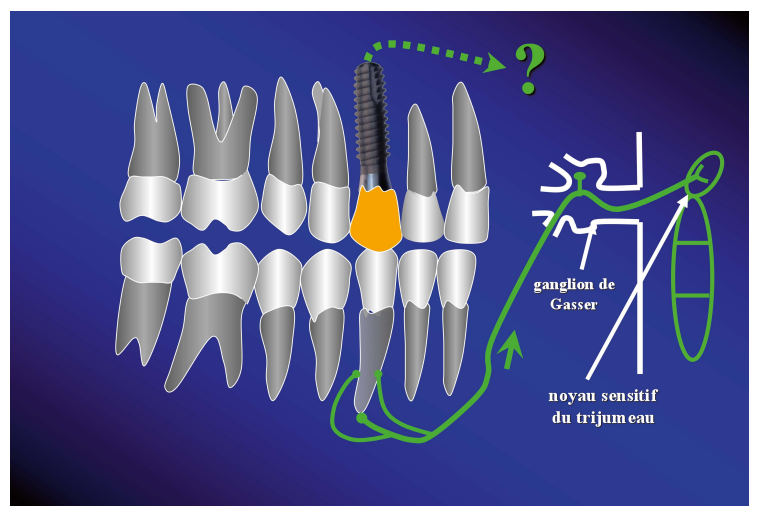

pas été choisi par le praticien et qui peut s'avérer être incorrect. Notons la définition du Petit Larousse 2010 au mot «Égression: Évolution d'une ou de plusieurs dents qui, n'ayant pas de dents antagonistes, quittent leur plan articulaire normal, paraissant ainsi sortir de leurs alvéoles ». Ce qui est su par le Larousse semble bien ignoré par de nombreux praticiens...;

I la fonction de groupe systématique. Théoriquement idéale, la fonction de groupe permet, en latéralité, de répartir les contraintes occlusales sur un nombre important de dents. On l'a vu plus haut, une fonction de groupe correcte est très difficile à réaliser, pour des raisons techniques, physiologiques et esthétiques. La plupart des auteurs prônant la fonction de groupe lorsque la canine est un implant ont oublié que l'occlusion est le rapport entre deux arcades dentaires, l'arcade maxillaire et l'arcade mandibulaire.

Lorsqu'un implant est en occlusion, il peut être face à : $\checkmark$ une dent, munie d'un desmodonte et de récepteurs desmodontaux, permettant au mécanisme neurophysiologique de protection d'être présent et d'éviter les surcharges occlusales (Fig. 21);

I une selle prothétique, ce sont alors les récepteurs de la muqueuse buccale, dont certains sont fins et discriminatifs, qui prennent le relais des récepteurs desmodontaux et permettent la mise en œuvre de la protection neurophysiologique (Fig. 22);

I un implant, et dans cette situation, il n'existe aucun mécanisme protecteur permettant d'éviter les surcharges occlusales (Fig. 23).

\section{LE CONCEPT OCCLUSAL DE LA CANINE SUR IMPLANT}

Le concept occluso-prothétique adopté doit être déterminé en fonction de l'absence ou de la présence de récepteurs desmodontaux, les seuls permettant de moduler la contraction de la musculature manducatrice et d'éviter les surcharges occlusales. Lorsque l'implant remplace une canine, la nature de l'arcade antagoniste devient essentielle :

$\checkmark$ l'élément antagoniste est une canine naturelle : c'est la meilleure situation possible, car ce sont les récepteurs desmodontaux de la canine antagoniste, qu'elle soit pulpée ou non, porteuse d'une prothèse ou non, qui guideront le mouvement, permettant la désocclusion immédiate des groupes cuspidés travaillants et non travaillants (Fig. 24).

Fig. 24 : Lorsque la canine implantée fait face à une canine naturelle, les récepteurs desmodontaux de cette canine naturelle informent les centres supérieurs des pressions reçues et peuvent moduler la contraction des muscles masticateurs. Le concept de la fonction canine peut être adopté car « neurophysiologiquement » cohérent. 

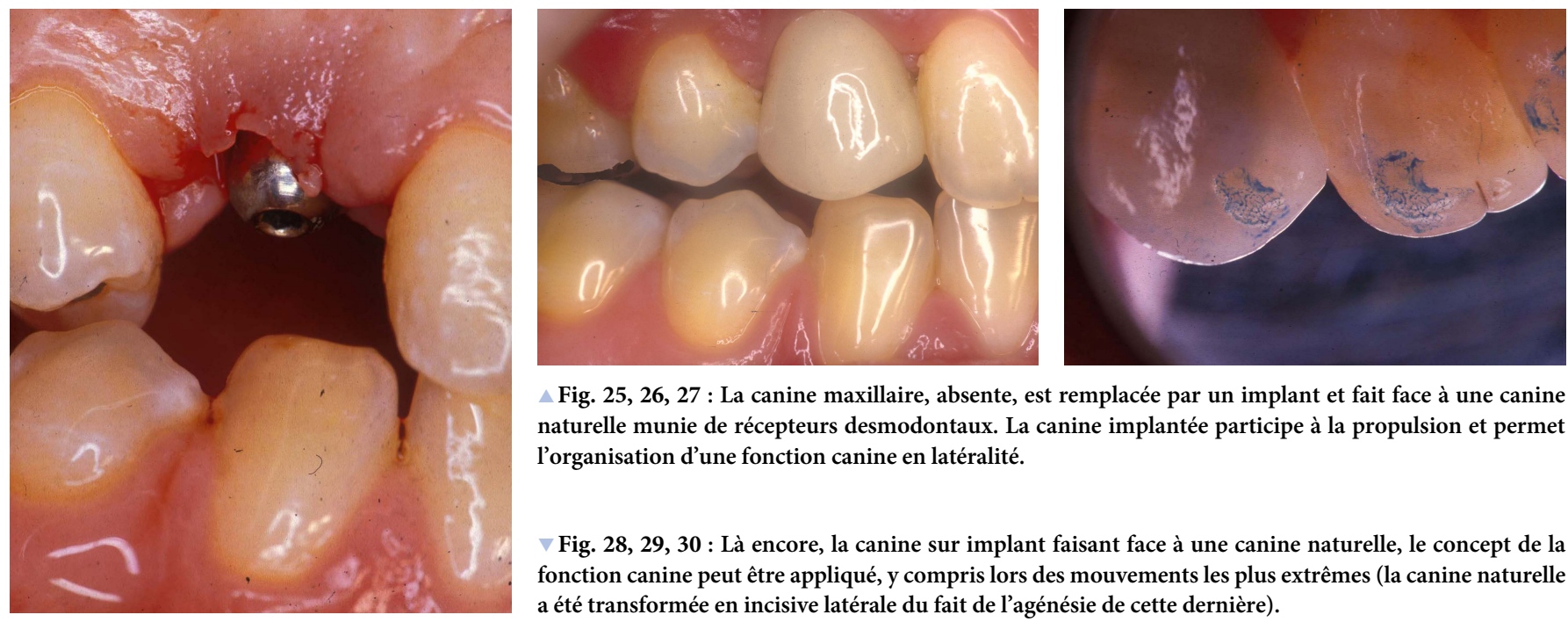

$\triangle$ Fig. 25, 26, 27 : La canine maxillaire, absente, est remplacée par un implant et fait face à une canine naturelle munie de récepteurs desmodontaux. La canine implantée participe à la propulsion et permet l'organisation d'une fonction canine en latéralité.

$\checkmark$ Fig. 28, 29, 30 : Là encore, la canine sur implant faisant face à une canine naturelle, le concept de la fonction canine peut être appliqué, y compris lors des mouvements les plus extrêmes (la canine naturelle a été transformée en incisive latérale du fait de l'agénésie de cette dernière).
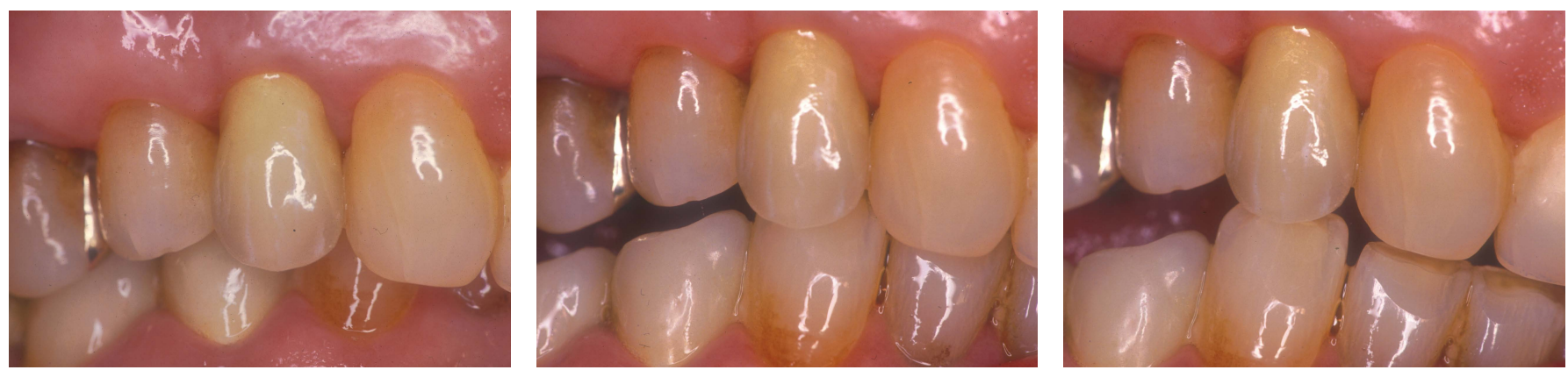

Une fonction canine impliquant un implant est alors parfaitement acceptable, la dent antagoniste permettant une protection d'origine neurophysiologique (Fig. 25 à 27, 28 à 30);

\- l'élément antagoniste est un implant : cette situation clinique est rare et n'a été rencontrée qu'une fois par l'auteur en plus de 35 années d'expérience. Elle doit être analysée logiquement, en fonction de la neurophysiologie des éléments présents (Fig. 31). La position d'intercuspidie maximale et la propulsion seront traitées classiquement car elles font intervenir, en plus des implants, des dents naturelles et leurs récepteurs desmodontaux. La latéralité travaillante

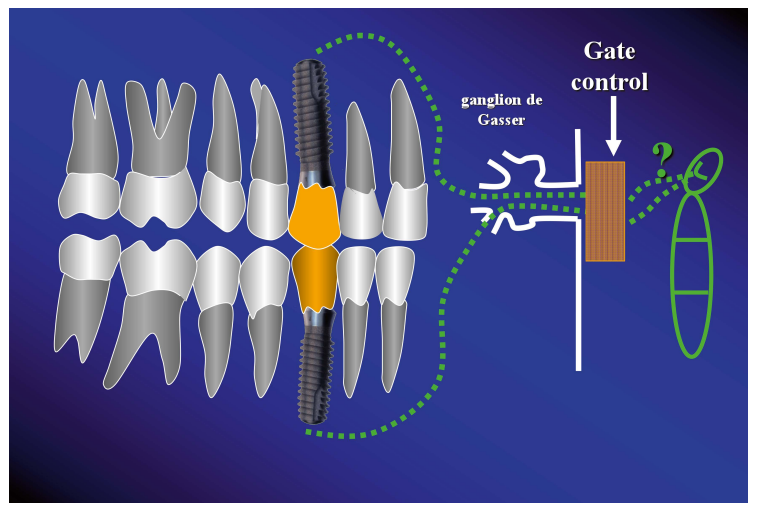

$\triangle$ Fig. 31 : Lorsque la canine maxillaire et la canine mandibulaire sont remplacées par des implants, il n'existe plus de récepteurs discriminatifs permettant de moduler la contraction de la musculature manducatrice en latéralité. faisant intervenir 2 implants antagonistes est totalement dépendante de la canine supérieure controlatérale :

Ila canine contro-latérale est courte et la latéralité peut se faire sous la forme d'une fonction de groupe «réduite », impliquant les dents bordant l'édentement : incisive latérale et première prémolaire au maxillaire et à la mandibule, avec une réduction de la hauteur des canines (Fig. 32).

Afin de pallier la difficulté de réalisation d'une fonction de groupe correcte, incluant toutes les positions possibles en latéralité, il faut adopter le concept de la désocclusion retardée des secteurs cuspidés proposée,

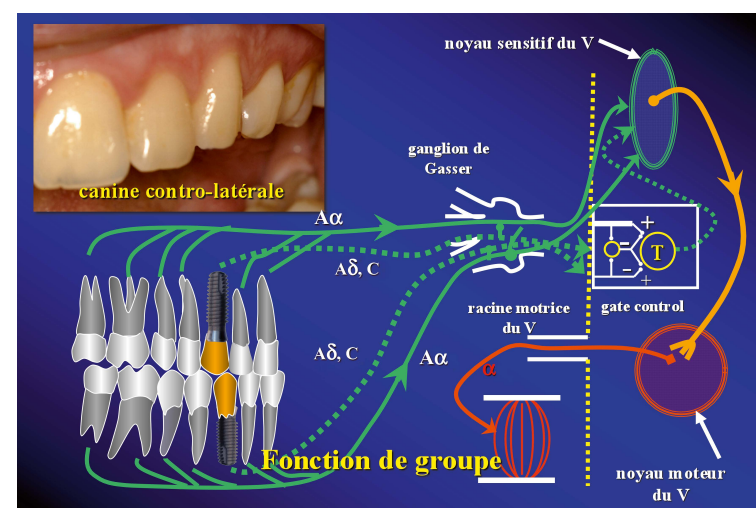

$\triangle$ Fig. 32 : En l'absence de récepteurs discriminatifs et lorsque la canine contro-latérale est courte, une fonction de groupe peut être envisagée, ce qui permet à des dents naturelles munies de récepteurs desmodontaux de participer à la latéralité du côté travaillant. 


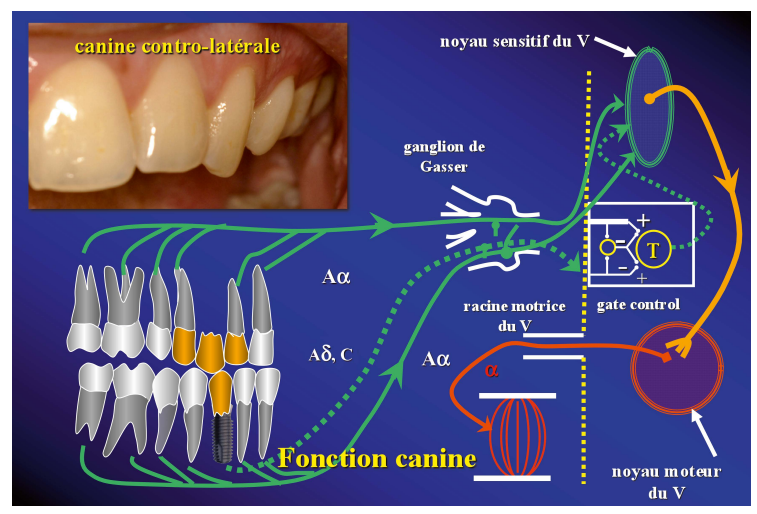

$\triangle$ Fig. 33 : Lorsque la canine maxillaire contro-latérale est longue et son " raccourcissement " esthétiquement impossible, la solution consiste à réaliser une prothèse fixe sur une des arcades. Cette prothèse, s'appuyant sur des piliers naturels, transmet des informations fines et discriminatives au noyau sensitif du trijumeau.
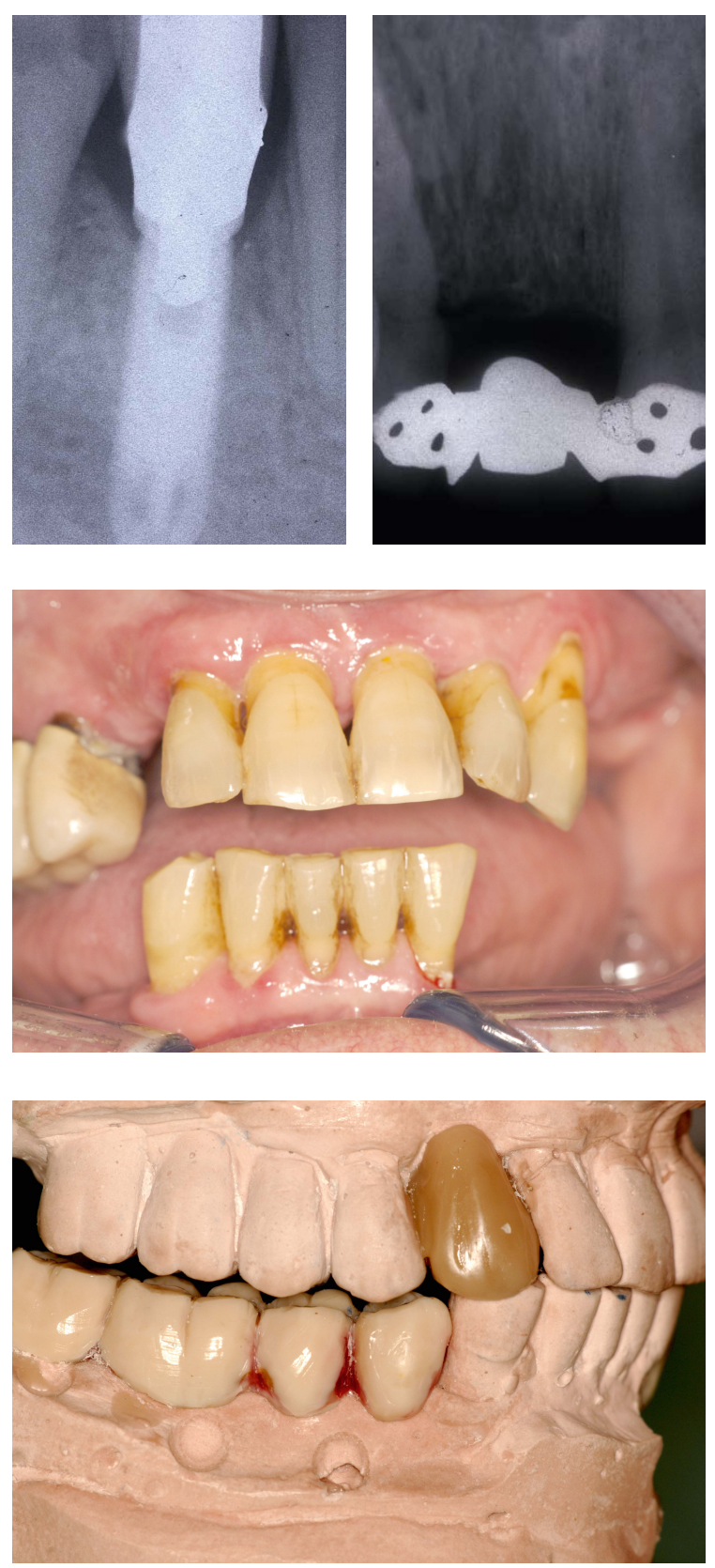

4 Fig. 36 : L'examen clinique montre la perte de la canine maxillaire droite et de la canine mandibulaire gauche. La canine maxillaire gauche et la canine mandibulaire droite sont présentes.

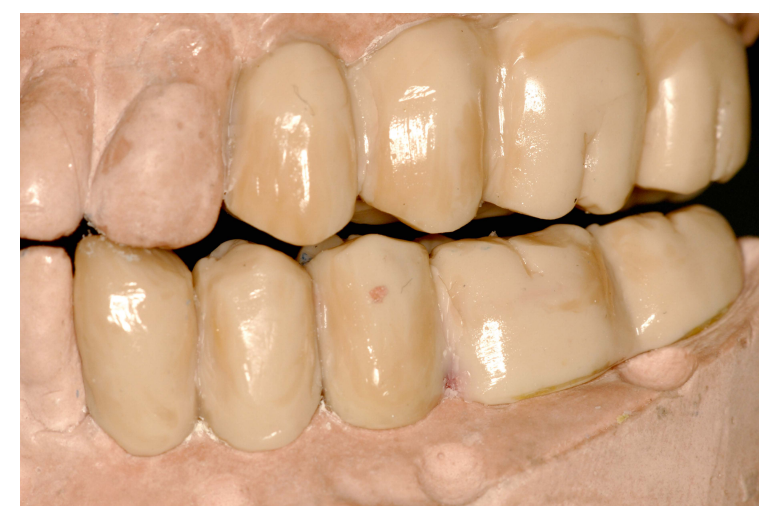

$\triangle$ Fig. 37 et 38 : Le choix du concept occlusal sera celui de la fonction canine, chaque canine implantée faisant face à une canine naturelle riche en récepteurs desmodontaux. entre autres, par Preiskkel dans les années 1980. Selon ce concept, les premiers millimètres de la trajectoire, enveloppe habituelle de la majorité des mouvements occlusaux, se font en fonction de groupe, puis, lorsque le mouvement s'amplifie et sort de son enveloppe habituelle, intervient une fonction canine. Ce concept permet de limiter le réglage des plans de glissement en latéralité aux premiers millimètres du mouvement, réduisant ainsi la difficulté de leur réalisation, puis, lorsque le mouvement s'amplifie, ce qui est en dehors de l'enveloppe habituelle des mouvements, donc rare, une fonction canine prend le relais. Il faut cependant bien noter que, même avec une désocclusion retardée et une fonction de groupe réduite à quelques dents, le raccourcissement de la canine maxillaire est indispensable, avec les conséquences esthétiques vues au paragraphe précédent;

I la canine contro-latérale est longue et l'indication de l'implant maxillaire doit être remise en cause au profit d'un bridge impliquant ou non une préparation pelliculaire pour un bridge collé ou une préparation périphérique des dents collatérales pour un bridge classique (Fig. 33, 34, 35).

$\measuredangle$ Fig. 34 et 35 : Dans ce cas unique de perte des canines maxillaire et mandibulaire droites, et la canine maxillaire gauche étant de longueur importante, l'option clinique choisie a fait intervenir un implant à la mandibule et un bridge collé au maxillaire. 
L'intermédiaire de prothèse remplaçant la canine maxillaire prend appui sur les dents naturelles bordant l'édentement, dents munies de récepteurs desmodontaux et permettant alors de moduler la contraction des muscles manducateurs.

En clinique, lorsque des canines sont absentes et doivent être remplacées par des implants, la recherche des récepteurs desmodontaux permet de s'orienter vers un concept occlusal réfléchi, avec à chaque fois, comme idée maîtresse, que le concept le plus simple sera le mieux réalisé (Fig. 36 à 38).

\section{CONCLUSION}

La connaissance de la neurophysiologie de l'appareil manducateur, basé en grande partie sur la présence de récepteurs desmodontaux, permet, en prothèse sur implant, d'orienter le choix du concept occlusal le mieux adapté au cas clinique considéré, en particulier lorsque une ou des canines sont remplacées par des implants.

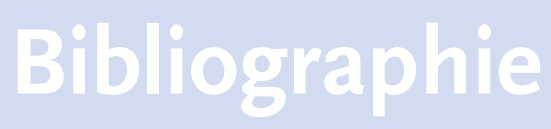

[1] Bert M. Les implants dentaires. CdP édit, Paris, 1987.

[2] Bert M, Missika P. Les clés du succès en implantologie. CdP édit, Rueil-Malmaison, 2009.

[3] Bert M. La canine en implantologie. Actual OdontoStomatol $2009 ; 245: 37-52$.

[4] Bert M., Leclercq Ph. L'occlusion en implantologie. EDP Sciences édit, Les Ulis, 2015.

[5] Higaki N., Goto T., Ishida Y., Watanabe M., Tomotake Y., Ichikawa T. Do sensation differences exist between dental implants and natural teeth ? A metaanalysis. Clin Oral Implants Res 2014; 25: 1307-1310.

[6] Mamo H. La douleur. Baillière édit, Paris, 1968.

[7] Noordenbos W. Pain. Elsevier édit, Amsterdam, 1959.

[8] Wall PD, Melzach R. Pain mechanism, a new theory. Science 1965;150:971-979.

[9] Woda A., Fontenelle A. Organisation générale du système nerveux. In Chateau M. ODF. Julien Prélat édit, Paris, 1975.

[10] Woda A. Abrégé de physiologie oro-faciale. Masson édit, Paris, 1983.

[11] Wolff J. Das gesetz der transformation der knochen. Hirschwald édit, Berlin, 1892. 